\title{
A cohort-based emergency plan to maintain functionality in a clinical laboratory during the 2020 COVID-19 epidemic outbreak
}

https://doi.org/10.1515/labmed-2020-0035

Received March 21, 2020; accepted May 9, 2020; previously published online June 2, 2020

\section{Abstract}

Objectives: Clinical laboratory analyses are essential part of critical care. Severe acute respiratory syndrome coronavirus 2 (SARS-CoV-2)/coronavirus disease 2019 (COVID19)-confirmed cases were doubling in Germany every 3 days during March 2020. Health care systems are preparing for an epidemic crisis.

Methods: We outline a cohort-based emergency planning. The plan is based on three independent self-sufficient cohorts that maintain duty for 7 days, followed by quarantine-like rest for 14 days. COVID-19-infected and otherwise ill personnel will be replaced by a tactical reserve, which is again replaced by recovered staff.

Results: We switched to the outlined system when incidence of confirmed COVID-19 cases surpassed 50/100,000 residents. Our parameter spectrum was reduced to the essential analyses in agreement with our clinical colleagues. So far the system works well. In model calculations, the system is robust to maintain essential laboratory functionality even when incidence of COVID-19 is higher than that currently observed in the most severely hit countries.

Conclusions: We outline a cohort-based emergency planning to maintain essential functionality of a clinical laboratory while minimizing the risk of spreading infection with COVID-19 among our workforce during the COVID-19 epidemic faced in 2020.

Keywords: emergency planning; laboratory management; SARS-CoV-2/Covid-19.

\footnotetext{
*Correspondence: Gerhard Pütz, University Medical Center Freiburg, Faculty of Medicine, Hugstetterstr. 55, 79106 Freiburg, Germany, E-Mail: gerhard.puetz@uniklinik-freiburg.de. https://orcid.org/0000-0002-0816-2018

Manuela Müller and Karl Winkler: University Medical Center Freiburg, Faculty of Medicine, Freiburg, Germany
}

\section{Background}

Severe acute respiratory syndrome coronavirus 2 (SARSCoV-2)/ coronavirus disease 2019 (COVID-19) is spreading fast in Western Europe, with exponential growth in several countries including Germany [1]. The University Medical Center Freiburg offers maximum care for about 1600 patients. Our laboratory usually provides essential clinical laboratory analysis on a 24/7 basis. While maintaining state-of-the-art hygienic standards, we may rule out the contamination of staff members by blood samples, but with the current exponential increase in active COVID-19 cases here in Freiburg and the region, we must now confront the risk of COVID-19 infection among our staff. Shutting down our central clinical laboratory unit for a 14-day quarantine as the authorities have proposed is no option, as we are obliged to ensure basic laboratory analysis essential for critical clinical care. On the other hand, uncontrolled dissemination among staff may lead to a forced shutdown because of the lack of healthy personnel able to continue working. Estimating a basic reproduction number $R_{0}$ of 2.2-3.6 [2] and a serial interval SI (the time to spread the infection) of 4.4-7.5 days [3], the uncontrolled spread of infection could incapacitate a clinical laboratory within 2 weeks. To maintain essential functionality while minimizing the risk of infection within the workforce, we implemented a cohort-based emergency plan that may enable laboratory functionality for a prolonged period of time. This emergency plan tries to take some basic principles into account:

- Use of independent self-sufficient cohorts, separated from each other

- Reliance on existing and well-established systems and workflow

- Sufficient personnel in a tactical reserve (TR)

- Flexibility

As several clinical laboratories in the affected areas may face similar problems, we hereby want to communicate our thoughts and developments. 
A
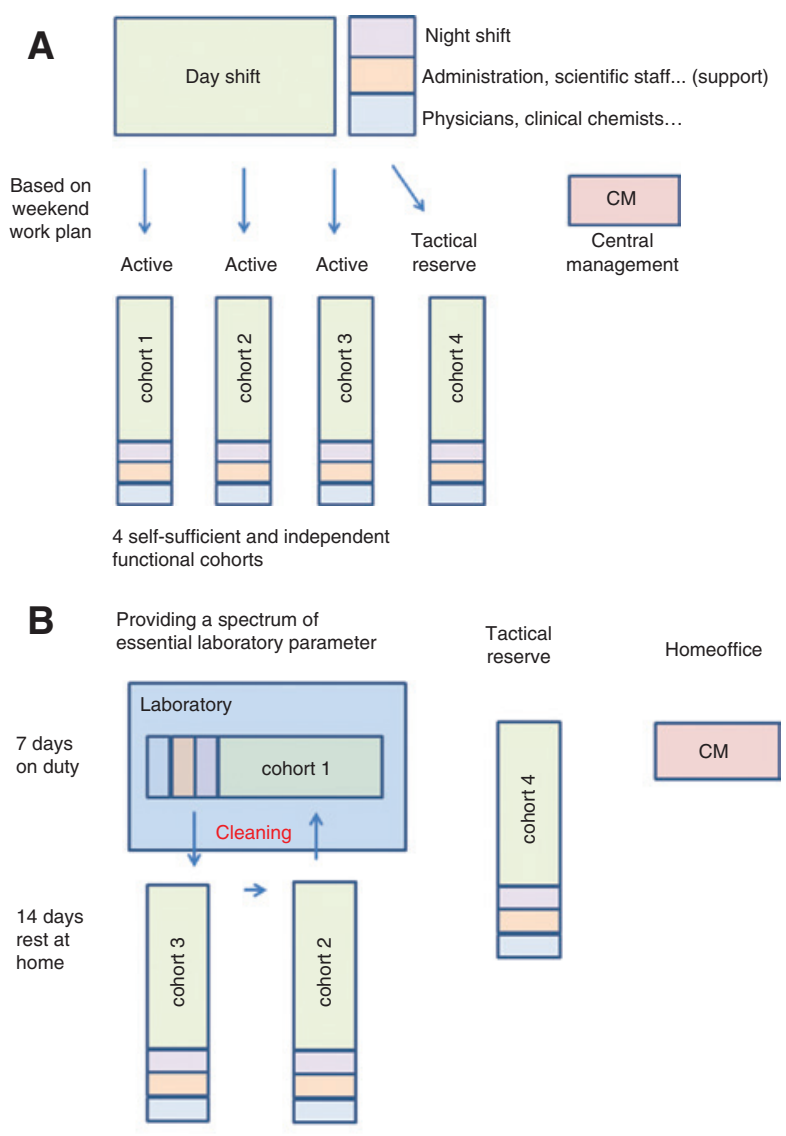

$\uparrow$ Change of shift after 7 days, cohorts must not mix!

\section{C}

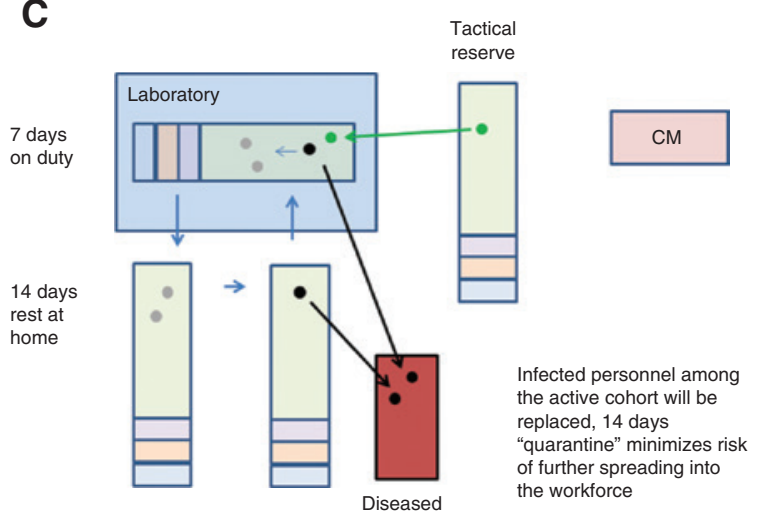

D

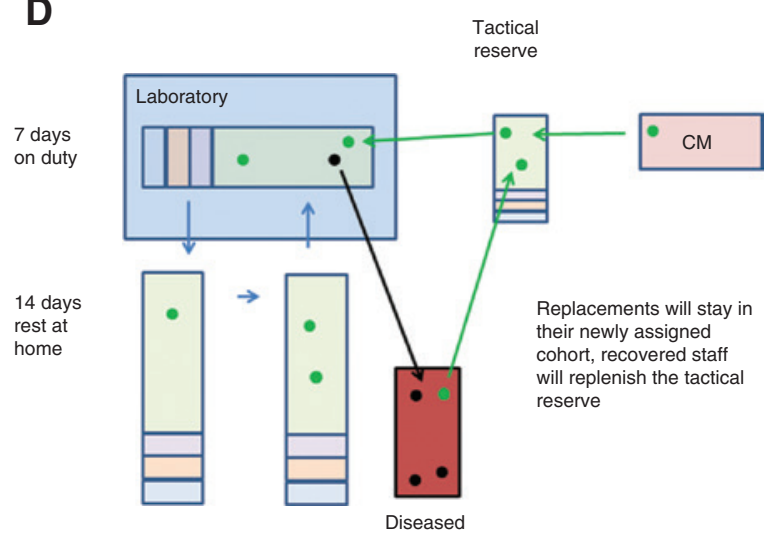

Figure 1: Schematic drawing of a cohort-based emergency plan to maintain essential laboratory functionality during the 2020 COVID-19 epidemic.

(A) Divide workforce into four self-sufficient independent cohorts.

(B) Three cohorts are active, one builds a tactical reserve. One cohort is on duty for 7 days, followed by a rest of 14 days. Important: cohorts never ever mix! (C) Infections are replaced by the tactical reserve, as well as other illness. Replacements stay in their assigned cohort. (D) Recovered personnel will replenish the tactical reserve and are assigned to a new cohort when necessary.

Outline for emergency planning in a clinical laboratory to maintain long-term functionality during a COVID-19 outbreak (Figure 1):

1. Limit activities to what is absolutely essential. Our list of essential parameters is based on those provided 24/7 during weekends and nighttime, with only few long-term essential parameters added.

2. Reduce the active workforce. Our basic duty roster resembles weekend scheduling, which is well known by the staff and is functionally tested.

3. Divide staff into four cohorts: three working and one TR, each working cohort must be able to function completely autonomous. Include physicians, clinical chemists and supporting personnel (like research staff, students, etc. into each cohort).

4. Basic rule: active cohorts must never, ever mix!!! Not even in private!

5. Each active cohort works for 7 continuous days (and nobody else is allowed in the lab during that shift!), followed by 14 days of rest. This ensures that COVID-19's dissemination within a cohort on duty does not spread through our entire staff, and the 14-day rest ensures a "quasi-quarantine" to stop a spread and may help to identify infected persons before they can spread the infection to their colleagues.

6. For shift changes between cohorts, the laboratory will be cleaned according to a rigid hygiene standard operating procedure (SOP) and evacuated completely by the old cohort prior to the new cohort's take-over. The shift leader will interview all personnel about health/infection risks.

7. Dropouts in the cohort on duty will be replaced by the backup cohort according to individual qualification, and remain in that cohort from that day on (never ever mixing!).

8. Recovered staff from any sick leave support the backup cohort (remembering to maintain 14 days off duty).

9. Once there are enough recovered personnel to form a new cohort (the "resistant cohort"), they may be regrouped and take over, hopefully joined by the rest of the unaffected staff soon. 


\section{Implementation and robustness}

As of 14.03.2020, about 70 cases of COVID-19 have been reported in the city of Freiburg and the surrounding hinterland, which has a population of approximately 500,000 residents. With 70 cases and an $R_{0}$ of 3.5 , we estimate about 250 potentially infected people among Freiburg residents, yielding a rate of $1 / 2000$ or a risk of $5 \times 10^{-4}$ for each staff member. As there are about 100 people working in our core laboratory, the risk that one of our staff members is currently infected cumulates to $5 \%$. In our opinion, this risk did not yet justify preventively switching to the emergency plan with its severely reduced service. However, considering this pandemic's dynamics, our risk assessment changed within few days. Six days later, on 19.03.2020, the confirmed cases in Freiburg had reached 248, raising the risk to $\sim 18 \%$ according to the rough estimation outlined earlier. Concurrently, the University Freiburg Medical Center was preparing for a wave of COVID-19 patients, closing down all outpatient care and elective surgeries. This led to a reduction in requests for laboratory analyses by $\sim 40 \%$. Thus, we decided to implement the outlined cohort system on 19.03.2020. The parameter spectrum was reduced in agreement with the clinical colleagues. Implementation worked well, and all staff was informed in person or via telephone by prepared telephone lists. So far, no significant problems occurred during the first 3 days, showing that in general the reduced workforce on duty is capable of running the laboratory properly.

To ensure long-term functionality, the balance between the rate of infection and rate of recoveries is crucial. From a recent study estimating the viral load in the respiratory system, about 20 days are necessary to become essentially negative. Thus, we assumed a time to recovery of 21 days [4]. At least in theory, over the long term, this system is likely robust up to $\sim 1 \%$ symptomatic cases in the general population (see Supplement), greatly exceeding the incidences of the COVID-19 pandemic experienced so far. Calculating the minimum head count of the TR depends on the workforce's flexibility, but we should be able to cope with a first hit and close by the second hit, taking out an estimated 10 staff members within short notice (see Supplement).

The system outlined earlier was designed for the specific circumstances at the University Medical Center Freiburg core laboratory unit on the eve of a COVID-19 epidemic. In general, it may be adapted to clinical or research labs or other workflows with a sufficient number of staff members. We hope that our considerations will help our valued colleagues with their very own preparations for what is to come.

Research funding: None declared.

Author contributions: All authors have accepted responsibility for the entire content of this manuscript and approved its submission.

Competing interests: Authors state no conflict of interest.

\section{References}

1. Dong E, Du H, Gardner L. An interactive web-based dashboard to track COVID-19 in real time. Lancet Infect Dis 2020;20:533-4.

2. Lai C-C, Shih T-P, Ko W-C, Tang H-J, Hsueh P-R. Severe acute respiratory syndrome coronavirus 2 (SARS-CoV-2) and coronavirus disease-2019 (COVID-19): the epidemic and the challenges. Int J Antimicrob Agents 2020;55:10592.

3. Anderson RM, Heesterbeek H, Klinkenberg D, Hollingsworth TD. How will country-based mitigation measures influence the course of the COVID-19 epidemic? Lancet 2020;395:931-4.

4. Zou L, Ruan F, Huang M, Liang L, Huang H, Hong Z, et al. SARSCoV-2 viral load in upper respiratory specimens of infected patients. N Engl J Med 2020;382:1177-9.

Supplementary Material: The online version of this article offers supplementary material (https://doi.org/10.1515/labmed-2020-0035). 